$10-8-2021$

\title{
On the Extension of Exponentiated Pareto Distribution
}

Amal S. Hassan

Cairo University, Giza, Egypt, dr.amalelmoslamy@gmail.com

Saeed Elsayed Hemeda

Obour Institutes, Cairo, Egypt, saidhemeda25jan@gmail.com

Said G. Nassr

Sinai University, Sinai, Egypt, dr.saidstat@gmail.com

Follow this and additional works at: https://digitalcommons.wayne.edu/jmasm

Part of the Applied Statistics Commons, Social and Behavioral Sciences Commons, and the Statistical Theory Commons

\section{Recommended Citation}

Hassan, Amal S.; Hemeda, Saeed Elsayed; and Nassr, Said G. (2021) "On the Extension of Exponentiated Pareto Distribution," Journal of Modern Applied Statistical Methods: Vol. 19 : Iss. 1 , Article 21. DOI: $10.22237 / \mathrm{jmasm} / 1619481840$

Available at: https://digitalcommons.wayne.edu/jmasm/vol19/iss1/21

This Regular Article is brought to you for free and open access by the Open Access Journals at DigitalCommons@WayneState. It has been accepted for inclusion in Journal of Modern Applied Statistical Methods by an authorized editor of DigitalCommons@WayneState. 


\section{On the Extension of Exponentiated Pareto Distribution}

\author{
Amal S. Hassan \\ Cairo University \\ Giza, Egypt
}

\author{
Saeed Elsayed Hemeda \\ Obour Institutes \\ Cairo, Egypt
}

\author{
Said G. Nassr \\ Sinai University \\ Sinai, Egypt
}

In this study, an extended exponentiated Pareto distribution is proposed. Some statistical properties are derived. We consider maximum likelihood, least squares, weighted least squares and Bayesian estimators. A simulation study is implemented for investigating the accuracy of different estimators. An application of the proposed distribution to a real data is presented.

Keywords: $\quad$ Exponentiated Pareto distribution, maximum likelihood estimation, least squares method, Bayesian method

\section{Introduction}

The Pareto distribution can be used quite effectively in analyzing many lifetime data. A two-parameter distribution, called the exponentiated Pareto (EP) distribution has been proposed by Gupta et al. (1998) as a simple generalization or modification of the well-known standard Pareto distribution by introducing one more shape parameter. The EP distribution can be decreasing and upside-down bathtub shaped failure rates. The probability density function (pdf) of the EP distribution is given by

$$
\mathrm{g}(x ; \lambda, \theta)=\lambda \theta(1+x)^{-(\lambda+1)}\left[1-(1+x)^{-\lambda}\right]^{\theta-1} ; \quad x, \lambda, \theta>0,
$$

where $\theta$ and $\lambda$ are two shape parameters. The cumulative distribution function (cdf) of EP distribution is given by

doi: 10.22237/jmasm/1619481840 | Accepted: Nov. 21, 2019; Published: October 8, 2021.

Correspondence: Said G. Nassr,dr.saidstat@gmail.com 


\section{HASSAN ET AL}

$$
\mathrm{G}(x ; \lambda, \theta)=\left[1-(1+x)^{-\lambda}\right]^{\theta} .
$$

Note that, if $\theta=1$, the EP distribution reduces to the standard Pareto (SP) distribution of the second kind.

In recent times, several generated classes of distributions were introduced and studied, by several authors. Among them, Marshall-Olkin-G (Marshall \& Olkin, 1997), beta-G (Eugene et al., 2002; Jones, 2004), gamma-G (Zografos \& Balakrishanan, 2009), Kumaraswamy-G (Cordeiro \& de Castro, 2011), generalized beta-G (Alexander et al., 2012), exponentiated generalized (Cordeiro et al., 2013), transformed-transformer (Alzaatreh et al., 2013), exponentiated $T-X$ (Alzaghal et al., 2013), Weibull-G (Bourguignon et al., 2014), Type-1 half-logistic (Cordeiro et al., 2015), logistic-X (Tahir et al., 2016), Kumaraswamy Weibull-G (Hassan \& Elgarhy, 2016b), exponentiated Weibull-G (Hassan \& Elgarhy, 2016a), additive Weibull-G (Hassan \& Hemeda, 2016), odd Lindley-G (Silva et al., 2017), Type II half logistic-G (Hassan, Elgarhy, \& Shakil, 2017), generalized additive Weibull-G (Hassan, Hemeda et al., 2017), generalized odd log-logistic-G (Cordeiro et al., 2017), power Lindley-G (Hassan \& Nassr, 2018b), inverse Weibull-G (Hassan \& Nassr, 2018a), odd Lomax-G (Cordeiro et al., 2019), Type II generalized ToppLeone-G (Hassan, Elgarhy, \& Ahmad, 2019), and extended odd Weibull-G (Alizadeh et al., 2019).

For a baseline continuous cdf $\mathrm{G}(x ; \xi)$, Cordeiro et al. (2013) defined the exponentiated generalized (EG) class of distributions by

$$
\mathrm{F}(x ; \alpha, \beta, \xi)=\left[1-(1-\mathrm{G}(x ; \xi))^{\alpha}\right]^{\beta},
$$

where $\alpha>0$ and $\beta>0$ are two shape parameters whose role is to govern skewness and generate distributions with heavier lighter tails. The pdf corresponding to (3) is given by

$$
\mathrm{f}(x ; \alpha, \beta, \xi)=\alpha \beta \mathrm{g}(x ; \xi)[1-\mathrm{G}(x ; \xi)]^{\alpha-1}\left[1-(1-\mathrm{G}(x ; \xi))^{\alpha}\right]^{\beta-1}
$$

where, $\mathrm{g}(x ; \xi)$ is the baseline pdf. Setting $\alpha=1$, the pdf (4) gives Lehmann Type I class. Also, for $\beta=1$, the pdf (4) gives the Lehmann Type II class. So, the family (4) generalizes both Lehmann Types I and II classes. Furthermore, the baseline distribution $\mathrm{G}(x ; \xi)$ is a special case of (4) when $\alpha=\beta=1$. 


\section{ON THE EXTENSION OF EXPONENTIATED PARETO DISTRIBUTION}

According to Cordeiro et al. (2013), the class of EG distributions shares an attractive physical interpretation whenever $\alpha$ and $\beta$ are positive integers. Consider a device made of independent components in a parallel system with each component is made of independent subcomponents identically distributed according to $\mathrm{G}(x ; \xi)$ in a series system. The device fails if all components fail and each component fails if any subcomponent fails. Let $X_{j 1}, \ldots, X_{j \alpha}$ denote the lifetimes of the subcomponents within the $j^{\text {th }}$ component, $j=1, \ldots, \beta$, with common cdf $\mathrm{G}(x ; \xi)$. Let $X_{j}$ denote the lifetime of the $j^{\text {th }}$ component and let $X$ denote the lifetime of the device. Therefore, the cdf of $X$ is as follows:

$$
\begin{aligned}
\mathrm{P}(X \leq x) & =\mathrm{P}\left(X_{1} \leq x, \ldots, X_{\beta} \leq x\right)=\mathrm{P}\left(X_{1} \leq x\right)^{\beta}=\left[1-\mathrm{P}\left(X_{1}>x\right)\right]^{\beta} \\
& =\left[1-\mathrm{P}\left(X_{11}>x, \ldots, X_{1 \alpha}>x\right)\right]^{\beta}=\left[1-\mathrm{P}\left(X_{11}>x\right)^{\alpha}\right]^{\beta} \\
& =\left[1-\left(1-\mathrm{P}\left(X_{11}<x\right)^{\alpha}\right)\right]^{\beta}
\end{aligned}
$$

and the lifetime of the device obeys the EG family of distributions. So, the goal of the present work is introducing a new extension for EP distribution based on the EG family with more flexibility than the baseline (2). The extensions of classical distributions sometimes provide reasonable parametric fits to particular applications as in lifetimes and reliability studies. The new distribution has more sub-models when compared with baseline distribution and hence it allows us to study more comprehensive structural properties.

\section{An Extended Exponentiated Pareto Distribution}

We introduce an extended form for EP distribution, named as exponentiated generalized exponentiated Pareto (EGEP) distribution. The four-parameter EGEP distribution is particular case from EG class presented by Cordeiro et al. (2013). Some sub-models and some related distributions are presented. Further, the reliability, hazard rate, cumulative hazard rate and odds ratio functions are given. Asymptotic and possible shapes of the pdf, cdf, and the mode of EGEP are discussed.

The cdf of the EGEP distribution is obtained by taking $\mathrm{G}(x ; \xi)$ to be cdf of EP distribution as follows: 


\section{HASSAN ET AL}

$$
\mathrm{F}(x ; \phi)=\left\{1-\left[1-\left(1-(1+x)^{-\lambda}\right)^{\theta}\right]^{\alpha}\right\}^{\beta} ; \quad x>0 ; \alpha, \beta, \lambda, \theta>0
$$

where $\phi=(\alpha, \beta, \lambda, \theta)$ is the set of parameters The pdf of EGEP distribution corresponding to (5) is written as follows:

$$
\begin{aligned}
f(x ; \phi)=\frac{\alpha \beta \lambda \theta}{(1+x)^{(\lambda+1)}}\left[1-(1+x)^{-\lambda}\right]^{\theta-1}\left[1-\left(1-(1+x)^{-\lambda}\right)^{\theta}\right]^{\alpha-1} \\
\times\left\{1-\left[1-\left(1-(1+x)^{-\lambda}\right)^{\theta}\right]^{\alpha}\right\}^{\beta-1}
\end{aligned}
$$

$X \sim \operatorname{EGEP}(\alpha, \beta, \lambda, \theta)$ denotes a random variable with the pdf (6). Figure 1 displays a variety of possible shapes of pdf of EGEP distribution for some selected values of parameters. Clearly, EGEP densities take various shapes such as unimodal, reversed J shaped, and right skewed.

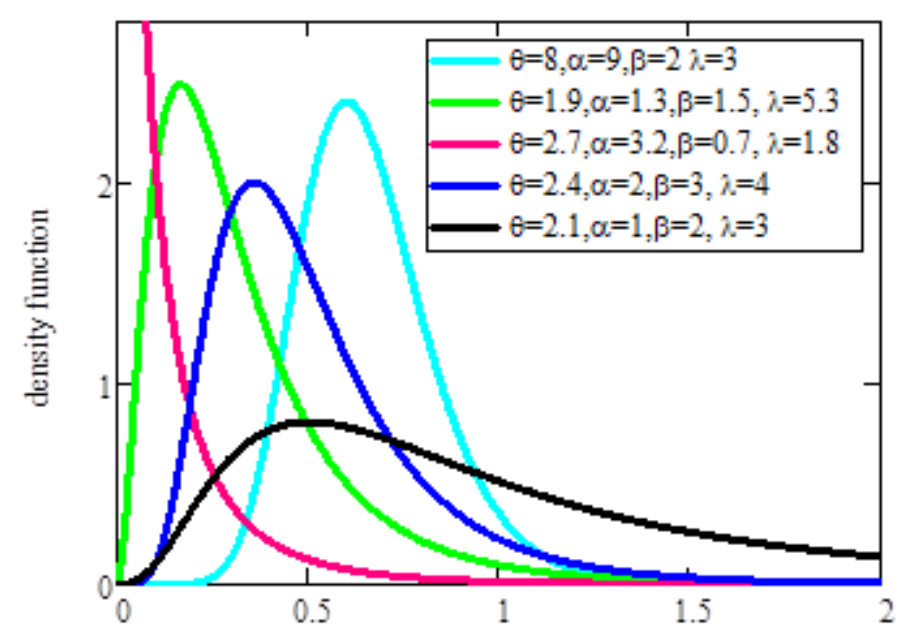

Figure 1. The pdf of EGEP distribution for different values of $\alpha, \beta, \lambda$, and $\theta$ 


\section{ON THE EXTENSION OF EXPONENTIATED PARETO DISTRIBUTION}

\section{Some Special Distributions}

Here, we present special distributions to EGEP model as follows:

- For $\beta=1$, the EGEP reduces to Generalized exponentiated Pareto (GEP) as a new model.

- For $\alpha=1$, the EGEP reduces to the Exponentiated Pareto (EP) distribution with power parameter $\theta \beta$.

- For $\alpha=1$ and $\beta=1$, the EGEP reduces to EP distribution (see Gupta et al., 1998).

- For $\alpha=1, \beta=1$, and $\theta=1$, the EGEP reduces to the Lomax model (see Lomax, 1954).

- For $\alpha=1, \beta=1, \lambda=1$, and $\theta=1$, the EGEP reduces to log-logistic model.

\section{Some Related Distributions}

Here, we provide some relations of the EGEP distribution to other distributions.

- For $Y=\ln (1+X), \quad X \sim \operatorname{EGEP}(\theta, \lambda, \alpha, \beta)$, the EGEP reduces to exponentiated Kumaraswamy exponential distribution (see Rodrigues \& Silva, 2015).

- For $Y=\ln (1+X), X \sim \operatorname{EGEP}(\theta, \lambda, 1,1)$, the EGEP distribution reduces to exponentiated exponential distribution (Gupta \& Kundu, 1999).

- For $Y=\ln (1+X), X \sim \operatorname{EGEP}(\theta, \lambda, \alpha, 1)$, the EGEP distribution reduces to Kumaraswamy exponential distribution (Cordeiro et al., 2010).

- For $Y=(1+X)^{-1}, X \sim \operatorname{EGEP}(\theta, \lambda, \alpha, \beta)$, the EGEP distribution reduces to Kumaraswamy Kumaraswamy distribution (El-Sherpieny \& Ahmed, 2014).

- For $Y=(1+X)^{-1}, X \sim \operatorname{EGEP}(\theta, \lambda, \alpha, 1)$, the EGEP distribution reduces to exponentiated Kumaraswamy distribution (Lemonte et al., 2013).

- For $Y=(1+X)^{-1}, X \sim \operatorname{EGEP}(\theta, \lambda, 1,1)$, the EGEP distribution reduces to Kumaraswamy distribution.

- For $Y=X / \delta, X \sim \operatorname{EGEP}(\theta, \lambda, 1,1)$, the $\operatorname{EGEP}$ distribution reduces to exponentiated Lomax distribution (Abdul-Moniem \& Abdel-Hameed, 2012).

\section{Reliability Analysis}

Expressions for the reliability function, hazard function, reversed hazard function, cumulative hazard rate and odds function are provided. 


\section{HASSAN ET AL}

The reliability function, denoted by $\overline{\mathrm{F}}(x ; \phi)$, is as follows:

$$
\overline{\mathrm{F}}(x ; \phi)=1-\left\{1-\left[1-\left(1-(1+x)^{-\lambda}\right)^{\theta}\right]^{\alpha}\right\}^{\beta} .
$$

The hazard rate function (hrf), say $\mathrm{h}(x ; \phi)$, is defined as follows:

$$
\begin{aligned}
\mathrm{h}(x ; \phi)=\frac{\alpha \beta \lambda \theta(1+x)^{-(\lambda+1)}\left[1-(1+x)^{-\lambda}\right]^{\theta-1}\left[1-\left(1-(1+x)^{-\lambda}\right)^{\theta}\right]^{\alpha-1}}{1-\left\{1-\left[1-\left(1-(1+x)^{-\lambda}\right)^{\theta}\right]^{\alpha}\right\}^{\beta}} \\
\times\left\{1-\left[1-\left(1-(1+x)^{-\lambda}\right)^{\theta}\right]^{\alpha}\right\}^{\beta-1}
\end{aligned}
$$

Figure 2 displays a variety of possible shapes of hrf of EGEP distribution for some selected values of parameters. It can be deduced from Figure 2 that the shape of the hazard function of the EGEP distribution could be constant, decreasing, and upside-down (depending on the value of the parameters).

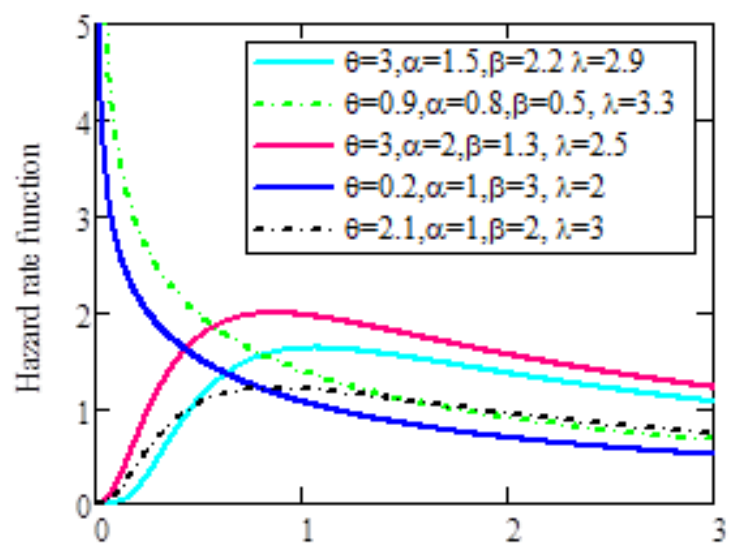

Figure 2. Hazard rate function of EGEP distribution for different values of $\alpha, \beta, \lambda$, and $\theta$ 


\section{ON THE EXTENSION OF EXPONENTIATED PARETO DISTRIBUTION}

Furthermore, the reversed hazard rate function, say $\mathrm{r}(x ; \phi)$; the cumulative hazard rate, say $\mathrm{H}(x ; \phi)$; and the odds function, say $\mathrm{O}(x ; \phi)$; are given, respectively, as follows:

$$
\begin{aligned}
& \mathrm{r}(x ; \phi)=\frac{\alpha \beta \lambda \theta(1+x)^{-(\lambda+1)}\left[1-(1+x)^{-\lambda}\right]^{\theta-1}\left[1-\left(1-(1+x)^{-\lambda}\right)^{\theta}\right]^{\alpha-1}}{1-\left(1-\left(1-(1+x)^{-\lambda}\right)^{\theta}\right)^{\alpha}}, \\
& \mathrm{H}(x ; \phi)=-\ln [\overline{\mathrm{F}}(x ; \phi)]=-\ln \left[1-\left\{1-\left[1-\left(1-(1+x)^{-\lambda}\right)^{\theta}\right]^{\alpha}\right\}^{\beta}\right],
\end{aligned}
$$

and

$$
\mathrm{O}(x ; \phi)=\left(\left\{1-\left[1-\left(1-(1+x)^{-\lambda}\right)^{\theta}\right]^{\alpha}\right\}^{-\beta}-1\right)^{-1}
$$

\section{Asymptotic \& Shapes}

We discuss the asymptotic and possible shapes of the cdf (5), pdf (6), reliability function (7), and hazard rate function (8) as follows:

i) When $x \rightarrow 0$,

$$
\operatorname{limf}_{x \rightarrow 0}(x ; \phi)=\lim _{x \rightarrow 0} \mathrm{~F}(x ; \phi)=\lim _{x \rightarrow 0} \mathrm{~h}(x ; \phi)=0, \text { and } \lim _{x \rightarrow 0} \overline{\mathrm{F}}(x ; \phi)=1 \text {. }
$$

ii) When $x \rightarrow \infty$,

$$
\lim _{x \rightarrow \infty} \mathrm{f}(x ; \phi)=\lim _{x \rightarrow \infty} \mathrm{F}(x ; \phi)=\lim _{x \rightarrow \infty} \mathrm{h}(x ; \phi)=0, \text { and } \lim _{x \rightarrow \infty} \overline{\mathrm{F}}(x ; \phi)=1 .
$$

\section{Mode of EGEP Distribution}

The mode $x_{0}$ of the EGEP distribution is obtained by solving the equation $\mathrm{f}^{\prime}(x)=0$, where 


\section{HASSAN ET AL}

$$
\begin{aligned}
& \mathrm{f}^{\prime}(x)=\alpha \beta \lambda \theta\left[\mathrm{A}^{\prime}(x) \mathrm{B}(x) \mathrm{C}(x) \mathrm{D}(x)+\mathrm{A}(x) \mathrm{B}^{\prime}(x) \mathrm{C}(x) \mathrm{D}(x)\right. \\
& \left.+\mathrm{A}(x) \mathrm{B}(x) \mathrm{C}^{\prime}(x) \mathrm{D}(x)+\mathrm{A}(x) \mathrm{B}(x) \mathrm{C}(x) \mathrm{D}^{\prime}(x)\right]=0
\end{aligned}
$$

where

$$
\begin{aligned}
& \mathrm{A}(x)=(1+x)^{-(\lambda+1)}, \mathrm{B}(x)=\left[1-(1+x)^{-\lambda}\right]^{\theta-1}, \mathrm{C}(x)=\left[1-\left(1-(1+x)^{-\lambda}\right)^{\theta}\right]^{\alpha-1}, \\
& \mathrm{D}(x)=\left[1-\left(1-\left(1-(1+x)^{-\lambda}\right)^{\theta}\right)^{\alpha}\right]^{\beta-1}, \mathrm{~A}^{\prime}(x)=-(\lambda+1)(1+x)^{-(\lambda+2)}, \\
& \mathrm{B}^{\prime}(x)=\lambda(\theta-1)(1+x)^{-(\lambda+1)}\left[1-(1+x)^{-\lambda}\right]^{\theta-2}, \\
& \mathrm{C}^{\prime}(x)=-\theta \lambda(\alpha-1)(1+x)^{-(\lambda+1)}\left[1-(1+x)^{-\lambda}\right]^{\theta-1}\left[1-\left(1-(1+x)^{-\lambda}\right)^{\theta}\right]^{\alpha-2}, \\
& \mathrm{D}^{\prime}(x)=\frac{\alpha \lambda \theta(\beta-1)}{(1+x)^{(\lambda+1)}}\left[1-(1+x)^{-\lambda}\right]^{\theta-1}\left[1-\left(1-(1+x)^{-\lambda}\right)^{\theta}\right]^{\alpha-1} \\
& \times\left[1-\left(1-\left(1-(1+x)^{-\lambda}\right)^{\theta}\right)^{\alpha}\right]^{\beta-2}
\end{aligned}
$$

Clearly, the root of the equation (9) is not available in closed form. So, numerical calculation is adopted to solve this equation for some values of parameters.

\section{Expansion for Density Function}

We consider the power series expansion, for any real non-integer $z$ :

$$
(1-z)^{\beta-1}=\sum_{k=0}^{\infty} \frac{(-1)^{k} \Gamma(\beta)}{\Gamma(\beta-k) k !} z^{k}
$$

which is valid for $|z|<1$. Then, by applying (10) in (6), the pdf of EGEP distribution, where $\beta$ is a real non-integer, becomes 


\section{ON THE EXTENSION OF EXPONENTIATED PARETO DISTRIBUTION}

$$
\begin{aligned}
f(x ; \phi)=\sum_{k=0}^{\infty} \frac{(-1)^{k} \alpha \lambda \theta \Gamma(\beta+1)}{\Gamma(\beta-k) k !(1+x)^{(\lambda+1)}}\left[1-(1+x)^{-\lambda}\right]^{\theta-1} & \\
& \times\left[1-\left(1-(1+x)^{-\lambda}\right)^{\theta}\right]^{\alpha+\alpha k-1}
\end{aligned}
$$

Again, using the binomial expansion (10) in (11),

$$
\mathrm{f}(x ; \phi)=\sum_{k, j=0}^{\infty} w_{k, j} \lambda \theta(1+x)^{-(\lambda+1)}\left[1-(1+x)^{-\lambda}\right]^{\theta+\theta j-1},
$$

where

$$
w_{k, j}=\frac{(-1)^{k+j} \alpha \beta \Gamma(\beta) \Gamma(\alpha+\alpha k)}{j ! \Gamma(\beta-k) \Gamma(\alpha+\alpha k-j) k !} .
$$

Further, (12) can be rewritten as follows:

$$
\mathrm{f}(x ; \phi)=\sum_{k, j=0}^{\infty} w_{k, j}^{*} \mathrm{~g}(x ; \lambda, \theta(j+1)),
$$

where

$$
w_{k, j}^{*}=\frac{w_{k, j}}{j+1}
$$

and $\mathrm{g}(x ; \lambda, \theta(j+1))$ is the pdf of EP distribution with parameters $\lambda$ and $\theta(j+1)$. Further, the cdf corresponding to (13) takes the following form:

$$
\mathrm{F}(x ; \phi)=\sum_{k, j=0}^{\infty} w_{k, j}^{*} \mathrm{G}(x ; \lambda, \theta(j+1)),
$$

where $\mathrm{G}(x ; \lambda, \theta(j+1))$ is the cdf of EP distribution with parameters $\lambda$ and $\theta(j+1)$. 


\section{HASSAN ET AL}

\section{Moments and Moments of the Residual Life}

Here, we derive the expression for ordinary and moments of residual life of EGEP distribution.

The $r^{\text {th }}$ moment for the EGEP is derived as follows:

$$
\mu_{r}^{\prime}=\int_{0}^{\infty} x^{r} \sum_{k, j=0}^{\infty} w_{k, j}^{*} \mathrm{~g}(x ; \lambda, \theta(j+1)) d x .
$$

Hence, after simplification, the $r^{\text {th }}$ moment for EGPF takes the form

$$
\mu_{r}^{\prime}=\sum_{k, j=0}^{\infty} \sum_{i=0}^{r}(-1)^{r-i}\left(\begin{array}{l}
r \\
i
\end{array}\right) w_{k, j}^{*} \theta(j+1) \mathrm{B}\left(1-\frac{i}{\lambda}, \theta(j+1)\right) .
$$

Some important measures, such as mean, variance, cumulants, skewness, and kurtosis, can be derived from (15). Setting $r=1$ and $r=2$ in (15), the mean and variance of EGEP are obtained as

$$
\mathrm{E}(X)=\sum_{k, j=0}^{\infty} w_{k, j}^{*}\left[\theta(j+1) \mathrm{B}\left(1-\frac{1}{\lambda}, \theta(j+1)\right)-1\right]
$$

and

$$
\begin{gathered}
\operatorname{Var}(X)=\sum_{k, j=0}^{\infty} w_{k, j}^{*}\left[1-2 \theta(j+1) \mathrm{B}\left(1-\frac{1}{\lambda}, \theta(j+1)\right)+\theta(j+1) \mathrm{B}\left(1-\frac{2}{\lambda}, \theta(j+1)\right)\right] \\
-\left\{\sum_{k, j=0}^{\infty} w_{k, j}^{*}\left[\theta(j+1) \mathrm{B}\left(1-\frac{1}{\lambda}, \theta(j+1)\right)-1\right]\right\}^{2}
\end{gathered}
$$

The residual life plays an important role in life testing situations and reliability theory. The $n^{\text {th }}$ moment of the residual life, $\mathrm{m}_{n}(t)=\mathrm{E}\left[(X-t)^{n} \mid X>t\right], n=1,2, \ldots$ uniquely determines $\mathrm{F}(x)$ (see Navarro et al., 1998). The $n^{\text {th }}$ moment of the residual life is defined by

$$
\mathrm{m}_{n}(t)=\frac{1}{\overline{\mathrm{F}}(t)} \int_{t}^{\infty}(x-t)^{n} \mathrm{f}(x) d x
$$




\section{ON THE EXTENSION OF EXPONENTIATED PARETO DISTRIBUTION}

Using pdf (13), the $n^{\text {th }}$ moment of the residual life of EGEP distribution is as follows:

$$
\mathrm{m}_{n}(t)=\frac{1}{\overline{\mathrm{F}}(t ; \phi)} \sum_{k, j=0}^{\infty} \sum_{r=0}^{n}(-1)^{n-r}\left(\begin{array}{l}
n \\
r
\end{array}\right) t^{n-r} w_{k, j}^{*} \int_{t}^{\infty} x^{r} \mathrm{~g}(x ; \lambda, \theta(j+1)) d x
$$

By using the binomial expansion and the upper incomplete gamma function, we obtain

$$
\begin{aligned}
\mathrm{m}_{n}(t)=\frac{1}{\overline{\mathrm{F}}(t ; \phi)} \sum_{k, j=0}^{\infty} \sum_{r=0}^{n} \sum_{h=0}^{r}\left[(-1)^{n-r}\left(\begin{array}{l}
n \\
r
\end{array}\right)\left(\begin{array}{l}
r \\
h
\end{array}\right) t^{n-r} w_{k, j}^{*} \lambda \theta(j+1)\right. & \\
& \left.\times \mathrm{B}\left((1+t)^{-\lambda}, 1-\frac{r}{\lambda}, \theta+\theta j\right)\right]
\end{aligned}
$$

where

$$
\mathrm{B}\left((1+t)^{-\lambda}, 1-\frac{r}{\lambda}, \theta+\theta j\right)
$$

is the incomplete beta function.

\section{Rényi Entropy}

For a certain random phenomenon under study, it is important to quantify the uncertainty associated with the random variable of interest. The Rényi entropy is one of the most popular measures used to quantify the variability of random variable $X$. The Rényi entropy of $X$ with density (6), say $\operatorname{IR}_{R}(\rho)$, for $\rho>0$ and $\rho \neq 1$ is given by

$$
\mathrm{I}_{\mathrm{R}}(\rho)=\frac{1}{1-\rho} \log \int_{-\infty}^{\infty} \mathrm{f}(x)^{\rho} d x
$$

Let 


\section{HASSAN ET AL}

$$
\begin{aligned}
\mathrm{f}(x ; \phi)^{\rho}=( & \alpha \beta \lambda \theta)^{\rho}(1+x)^{-\rho(\lambda+1)}\left[1-(1+x)^{-\lambda}\right]^{\rho(\theta-1)} \\
& \times\left[1-\left(1-(1+x)^{-\lambda}\right)^{\theta}\right]^{\rho(\alpha-1)}\left\{1-\left[1-\left(1-(1+x)^{-\lambda}\right)^{\theta}\right]^{\alpha}\right\}^{\rho(\beta-1)}
\end{aligned}
$$

Using the binomial expansion (10) twice in (16), we obtain

$$
\mathrm{f}(x ; \phi)^{\rho}=(\alpha \beta \lambda \theta)^{\rho} \sum_{k, j=0}^{\infty} \xi_{k, j}(1+x)^{-\rho(\lambda+1)}\left[1-(1+x)^{-\lambda}\right]^{\rho(\theta-1)+\theta j},
$$

where

$$
\xi_{k, j}=\frac{(-1)^{k+j}(\rho(\beta-1)) !(\rho(\alpha-1)+\alpha k) !}{(\rho(\beta-1)-k) !(\rho(\alpha-1)+\alpha k-j) ! k ! j !} .
$$

Hence, after some simplification, we have Rényi entropy as follows:

$$
\mathrm{I}_{\mathrm{R}}(\rho)=\frac{1}{1-\rho} \log \left[\sum_{k, j=0}^{\infty} \xi_{k, j} \int_{0}^{\infty}(\alpha \beta \lambda \theta)^{\rho}(1+x)^{-\rho(\lambda+1)}\left[1-(1+x)^{-\lambda}\right]^{\rho(\theta-1)+\theta j} d x\right] .
$$

Hence, the Rényi entropy of $X$ can be expressed as follows:

$$
\mathrm{I}_{\mathrm{R}}(\rho)=\frac{1}{1-\rho} \log \left[\sum_{k, j=0}^{\infty} \xi_{k, j}(\alpha \beta \theta)^{\rho} \lambda^{\rho-1} \mathrm{~B}\left(\frac{\rho(\lambda+1)-1}{\lambda}, \rho(\theta-1)+\theta j+1\right)\right] .
$$

\section{Parameter Estimation}

The estimators of the EGEP model parameters are obtained using maximum likelihood (ML), least squares (LS), weighted least squares (WLS) and Bayesian methods. 


\section{ON THE EXTENSION OF EXPONENTIATED PARETO DISTRIBUTION}

\section{Maximum Likelihood Estimators}

Let $X_{1}, X_{2}, \ldots, X_{n}$ be a simple random sample from the EGEP distribution with set of parameters $\phi=(\alpha, \lambda, \theta, \beta)$. The likelihood function based on the observed random sample of size $\mathrm{n}$ from density (6) is given by

$$
\mathrm{L}(\phi \mid x)=(\alpha \beta \lambda \theta)^{n} \prod_{i=1}^{n} S_{i}^{-(\lambda+1)} Q_{i}^{\theta-1}\left[1-Q_{i}^{\theta}\right]^{\alpha-1}\left\{1-\left[1-Q_{i}^{\theta}\right]^{\alpha}\right\}^{\beta-1},
$$

where $S_{i}=\left(1+x_{i}\right)$ and $Q_{i}=\left(1-S_{i}^{-\lambda}\right)$. The natural logarithm likelihood function, denoted by $\ln l$, is obtained as follows:

$$
\begin{aligned}
\ln l=n \ln \alpha+n \ln \beta+n & \ln \lambda+n \ln \theta-(\lambda+1) \sum_{i=1}^{n} \ln S_{i}+(\theta-1) \sum_{i=1}^{n} \ln \left(Q_{i}\right) \\
& +(\alpha-1) \sum_{i=1}^{n} \ln \left[1-Q_{i}^{\theta}\right]+(\beta-1) \sum_{i=1}^{n} \ln \left\{1-\left[1-Q_{i}^{\theta}\right]^{\alpha}\right\}
\end{aligned}
$$

The partial derivatives of the log-likelihood function with respect to the unknown parameters are given by

$$
\begin{gathered}
\frac{\partial \ln l}{\partial \lambda}=\frac{n}{\lambda}-\sum_{i=1}^{n} \ln S_{i}+(\theta-1) \sum_{i=1}^{n} Q_{i}^{-1} S_{i}^{-\lambda} \ln S_{i}-\theta(\alpha-1) \sum_{i=1}^{n}\left[1-Q_{i}^{\theta}\right]^{-1} Q_{i}^{\theta-1} S_{i}^{-\lambda} \ln S_{i} \\
+\alpha \theta(\beta-1) \sum_{i=1}^{n}\left\{1-\left[1-Q_{i}^{\theta}\right]^{\alpha}\right\}^{-1}\left[1-Q_{i}^{\theta}\right]^{\alpha-1} Q_{i}^{\theta-1} S_{i}^{-\lambda} \ln S_{i}, \\
\frac{\partial \ln l}{\partial \alpha}=\frac{n}{\alpha}+\sum_{i=1}^{n} \ln \left[1-Q_{i}^{\theta}\right]-(\beta-1) \sum_{i=1}^{n}\left\{1-\left[1-Q_{i}^{\theta}\right]^{\alpha}\right\}^{-1}\left[1-Q_{i}^{\theta}\right]^{\alpha} \ln \left[1-Q_{i}^{\theta}\right], \\
\begin{array}{c}
\frac{\partial \ln l}{\partial \theta}=\frac{n}{\theta}+\sum_{i=1}^{n} \ln \left(Q_{i}\right)-(\alpha-1) \sum_{i=1}^{n}\left[1-Q_{i}^{\theta}\right]^{-1} Q_{i}^{\theta} \ln \left(Q_{i}\right) \\
+\alpha(\beta-1) \sum_{i=1}^{n} \ln \left\{1-\left[1-Q_{i}^{\theta}\right]^{\alpha}\right\}^{-1}\left[1-Q_{i}^{\theta}\right]^{\alpha-1} Q_{i}^{\theta} \ln \left(Q_{i}\right),
\end{array}
\end{gathered}
$$

and

$$
\frac{\partial \ln l}{\partial \beta}=\frac{n}{\beta}+\sum_{i=1}^{n} \ln \left\{1-\left[1-Q_{i}^{\theta}\right]^{\alpha}\right\}
$$




\section{HASSAN ET AL}

The ML estimators of the model parameters are determined by solving numerically the non-linear equations $\partial \ln l / \partial \alpha=0, \partial \ln l / \partial \lambda=0, \partial \ln l / \partial \theta=0$, and $\partial \ln l / \partial \beta=0$ simultaneously.

\section{Least Squares and Weighted Least Squares Estimators}

Suppose $X_{1}, X_{2}, \ldots, X_{n}$ is a random sample of size $\mathrm{n}$ from EGEP distribution and suppose $X_{1: n}, X_{2: n}, \ldots, X_{n: n}$ denotes the corresponding ordered sample. According to Johnson et al. (1995), the expectation and the variance of distribution are independent of the unknown parameter and are given by

$$
\mathrm{E}\left[\mathrm{F}\left(X_{i: n}\right)\right]=\frac{i}{n+1} \quad \text { and } \quad \operatorname{Var}\left[\mathrm{F}\left(X_{i: n}\right)\right]=\frac{i(n-i+1)}{(n+1)^{2}(n+2)}
$$

where $\left[\mathrm{F}\left(X_{i: n}\right)\right]$ is the cdf for any distribution and $X_{i: n}$ is the $i^{\text {th }}$ order statistic. Then LS estimators can be obtained by minimizing the sum of squares errors,

$$
\sum_{i=1}^{n}\left[\mathrm{~F}\left(X_{i: n}\right)-\frac{i}{n+1}\right]^{2}
$$

with respect to the unknown parameters. So, the LS estimators of the unknown parameters $\alpha, \lambda, \theta$, and $\beta$ of the EGEP model can be obtained by minimizing the following quantity:

$$
\sum_{i=1}^{n}\left[\left\{1-\left[1-\left(1-\left(1+x_{i: n}\right)^{-\lambda}\right)^{\theta}\right]^{\alpha}\right\}^{\beta}-\frac{i}{n+1}\right]^{2}
$$

with respect to $\alpha, \lambda, \theta$, and $\beta$. WLS estimators can be obtained by minimizing the sum of squares errors

$$
\sum_{i=1}^{n} \frac{1}{\operatorname{Var}\left(\mathrm{F}\left(X_{i: n}\right)\right)}\left[\mathrm{F}\left(X_{i: n}\right)-\mathrm{E}\left(\mathrm{F}\left(X_{i: n}\right)\right)\right]^{2}
$$

with respect to the unknown parameters $\alpha, \lambda, \theta$, and $\beta$. Therefore, the WLS estimators can be obtained by minimizing the following quantity 


\section{ON THE EXTENSION OF EXPONENTIATED PARETO DISTRIBUTION}

$$
\sum_{i=1}^{n} \frac{(n+1)^{2}(n+2)}{i(n-i+1)}\left[\left\{1-\left[1-\left(1-\left(1+x_{i: n}\right)^{-\lambda}\right)^{\theta}\right]^{\alpha}\right\}^{\beta}-\frac{i}{n+1}\right]^{2}
$$

with respect to the parameters $\alpha, \lambda, \theta$, and $\beta$.

\section{Bayesian Estimation and MCMC Approach}

The Bayesian estimator using squared error loss (SEL) function under the assumption of uniform priors of the population parameters for EGEP distribution is obtained. We consider the Bayesian estimation (BE) under the assumption that the random variables $\alpha, \lambda, \theta$, and $\beta$ independently distributed with non-informative type of priors

$$
\mathrm{g}(\alpha) \propto \alpha^{-1}, \mathrm{~g}(\lambda) \propto \lambda^{-1}, \mathrm{~g}(\beta) \propto \beta^{-1} \text {, and } \mathrm{g}(\theta) \propto \theta^{-1}
$$

Hence, the joint prior pdf of the unknown parameters can be expressed by

$$
\pi^{*}(\alpha, \lambda, \beta, \theta) \propto(\alpha \lambda \beta \theta)^{-1}
$$

Combining (17) and (18) to obtain the posterior density of $\phi=(\alpha, \lambda, \theta, \beta)$ given the data as follows:

$$
\begin{aligned}
\pi(\phi \mid x) \propto(\alpha \beta \lambda \theta)^{n-1} \prod_{i=1}^{n} S_{i}^{-(\lambda+1)}\left(1-S_{i}^{-\lambda}\right)^{\theta-1} & \left.1-\left(1-S_{i}^{-\lambda}\right)^{\theta}\right]^{\alpha-1} \\
& \times\left\{1-\left[1-\left(1-S_{i}^{-\lambda}\right)^{\theta}\right]^{\alpha}\right\}^{\beta-1}
\end{aligned}
$$

Therefore, the Bayesian estimator of parameters, say $\phi=(\alpha, \lambda, \beta, \theta)$ under SEL function; denoted by $\tilde{\mathrm{u}}_{(\mathrm{SEL})}(\phi)$ can be calculated through the following equations as follows:

$$
\tilde{\mathrm{u}}_{(\mathrm{SEL})}(\phi)=\mathrm{E}(\phi \mid \underline{x})=\int_{0}^{\infty} \int_{0}^{\infty} \int_{0}^{\infty} \int_{0}^{\infty} \phi \mathrm{L}(\phi \mid x) \pi(\phi \mid x) d \alpha d \lambda d \theta d \beta
$$




\section{HASSAN ET AL}

Generally, the ratio of four integrals given by equations (19) and (20) cannot be obtained in a closed form. In this case, we use the MCMC technique to generate samples from the posterior distributions and then compute the Bayesian estimators of the individual parameters. The conditional posterior densities of $\alpha, \lambda, \theta$, and $\beta$ are as follows:

$$
\begin{aligned}
& \pi_{1}(\alpha \mid x) \propto \alpha^{n-2} \prod_{i=1}^{n}\left[1-\left(1-S_{i}^{-\lambda}\right)^{\theta}\right]^{\alpha-1}\left\{1-\left[1-\left(1-S_{i}^{-\lambda}\right)^{\theta}\right]^{\alpha}\right\}^{\beta-1} \\
& \pi_{2}(\lambda \mid x) \propto \lambda^{n-2} \prod_{i=1}^{n} S_{i}^{-(\lambda+1)}\left(1-S_{i}^{-\lambda}\right)^{\theta-1}\left[1-\left(1-S_{i}^{-\lambda}\right)^{\theta}\right]^{\alpha-1}\left\{1-\left[1-\left(1-S_{i}^{-\lambda}\right)^{\theta}\right]^{\alpha}\right\}^{\beta-1} \\
& \pi_{3}(\theta \mid x) \propto \theta^{n-2} \prod_{i=1}^{n}\left(1-S_{i}^{-\lambda}\right)^{\theta-1}\left[1-\left(1-S_{i}^{-\lambda}\right)^{\theta}\right]^{\alpha-1}\left\{1-\left[1-\left(1-S_{i}^{-\lambda}\right)^{\theta}\right]^{\alpha}\right\}^{\beta-1}
\end{aligned}
$$

and

$$
\pi_{4}(\beta \mid x) \propto \beta^{n-2} \prod_{i=1}^{n}\left\{1-\left[1-\left(1-S_{i}^{-\lambda}\right)^{\theta}\right]^{\alpha}\right\}^{\beta-1}
$$

The Bayesian estimators are computed using the idea of Markov Chain Monte Carlo (MCMC) method based on Gibbs sampling. Therefore, to generate from this distribution, we use the Metropolis-Hastings method. To run the Gibbs sampler algorithm, we start with the ML estimates. We then draw samples from various full conditionals, in run, using the most recent values of all other conditioning variables unless some systematic pattern of convergence is achieved.

\section{Gibbs Sampling Algorithm}

The algorithm of Gibbs sampling is described as follows:

Step 1: $\quad$ Start with $\left(\alpha^{(0)}=\alpha, \lambda^{(0)}=\lambda, \theta^{(0)}=\theta, \beta^{(0)}=\beta\right)$ and set $I=1$.

Step 2: Generate $\alpha^{I}$ from $\pi_{1}(\alpha \mid x)$.

Step 3: Generate $\lambda^{I}$ from $\pi_{2}(\lambda \mid x)$.

Step 4: Generate $\theta^{I}$ from $\pi_{3}(\theta \mid x)$.

Step 5: Generate $\beta^{I}$ from $\pi_{4}(\beta \mid x)$.

Step 6: Compute $\alpha^{I}, \lambda^{I}, \theta^{I}$, and $\beta^{I}$.

Step 7: $\quad$ Set $I=I+1$.

Step 8: Repeat steps 2-6 $N$ times. 


\section{ON THE EXTENSION OF EXPONENTIATED PARETO DISTRIBUTION}

$$
\begin{aligned}
& \text { Step 9: We obtain the Bayes MCMC point estimate of } \\
& \phi_{q} \equiv\left(\phi_{1}=\alpha, \phi_{2}=\lambda, \phi_{3}=\theta, \phi_{4}=\beta\right), q=1,2,3 \text {, and } 4 \text { as } \\
& \qquad \mathrm{E}\left(\phi_{q} \mid \text { data }\right) \propto \frac{1}{N-M} \sum_{i=M+1}^{N} \phi_{q}^{(i)}
\end{aligned}
$$

where $M$ is the burn-in period (that is, a number of iterations before the stationary distribution is achieved) and the posterior variance of $\phi$ becomes

$$
\operatorname{Var}\left(\phi_{q} \mid \text { data }\right) \propto \frac{1}{N-M} \sum_{i=M+1}^{N}\left[\phi_{q}^{(i)}-\hat{\mathrm{E}}\left(\phi_{q} \mid \text { data }\right)\right]^{2}
$$

\section{Simulation Study}

The derived expressions for the estimators are too complicated to study analytically. Consequently, a numerical study is performed to compare the different estimators. The performances of the different estimates are compared in terms of their mean square error (MSE) and standard error (SE). The numerical procedure is described below:

- 10000 random samples of sizes 10, 20, 30, and 50 are generated from the EGEP distribution.

- Four sets of parameter values are selected as:

Case $\mathrm{I} \equiv(\alpha=0.4, \lambda=0.7, \theta=0.25, \beta=1.2)$,

Case II $\equiv(\alpha=0.5, \lambda=0.7, \theta=0.25, \beta=1.5)$,

Case III $\equiv(\alpha=1, \lambda=2.6, \theta=2, \beta=0.75)$, and

Case IV $\equiv(\alpha=0.4, \lambda=0.5, \theta=0.7, \beta=0.75)$,

- The ML, LS and WLS estimates of the unknown parameters are obtained.

- MSEs and SEs of different estimates of unknown parameters are computed. 


\section{HASSAN ET AL}

Table 1. MSEs, SEs, and MC errors of EGEP estimates for Case I and Case II

\begin{tabular}{|c|c|c|c|c|c|c|c|c|c|c|}
\hline \multirow[b]{2}{*}{$n$} & \multirow[b]{2}{*}{ Method } & \multirow[b]{2}{*}{ Properties } & \multicolumn{4}{|c|}{ Case I } & \multicolumn{4}{|c|}{ Case II } \\
\hline & & & $\alpha=0.4$ & $\lambda=0.7$ & $\theta=0.25$ & $\beta=1.2$ & $\alpha=0.5$ & $\lambda=0.7$ & $\theta=0.25$ & $\beta=1.5$ \\
\hline \multirow[t]{9}{*}{10} & $\mathrm{ML}$ & MSE & 1.697 & 1.149 & 0.630 & 0.608 & 0.763 & 1.419 & 1.016 & 1.811 \\
\hline & & SE & 0.121 & 0.101 & 0.076 & 0.075 & 0.083 & 0.108 & 0.095 & 0.130 \\
\hline & LS & MSE & 0.116 & 0.492 & 0.075 & 0.230 & 0.136 & 0.624 & 0.134 & 0.481 \\
\hline & & SE & 0.034 & 0.064 & 0.024 & 0.042 & 0.037 & 0.071 & 0.033 & 0.063 \\
\hline & WLS & MSE & 0.122 & 0.556 & 0.076 & 0.206 & 0.135 & 0.741 & 0.156 & 0.495 \\
\hline & & SE & 0.035 & 0.068 & 0.025 & 0.040 & 0.037 & 0.076 & 0.036 & 0.065 \\
\hline & $\mathrm{BE}$ & MSE & 0.344 & 0.594 & 0.535 & 0.333 & 0.202 & 0.555 & 0.480 & 0.794 \\
\hline & & SE & 0.005 & 0.049 & $9.300^{*}$ & 0.021 & 0.003 & 0.047 & $8.900^{*}$ & 0.061 \\
\hline & & MC Error & $1.134^{*}$ & 0.015 & $2.803^{*}$ & $6.411^{*}$ & $0.888^{*}$ & 0.015 & $2.735^{\star}$ & 0.019 \\
\hline \multirow[t]{9}{*}{20} & ML & MSE & 0.702 & 0.704 & 0.078 & 0.188 & 0.429 & 0.792 & 0.274 & 0.903 \\
\hline & & SE & 0.039 & 0.040 & 0.013 & 0.022 & 0.032 & 0.041 & 0.024 & 0.048 \\
\hline & LS & MSE & 0.059 & 0.327 & 0.045 & 0.141 & 0.062 & 0.449 & 0.081 & 0.347 \\
\hline & & SE & 0.012 & 0.026 & $9.044^{*}$ & 0.013 & 0.120 & 0.029 & 0.013 & 0.024 \\
\hline & WLS & MSE & 0.080 & 0.361 & 0.060 & 0.146 & 0.061 & 0.529 & 0.085 & 0.358 \\
\hline & & SE & 0.014 & 0.027 & 0.011 & 0.014 & 0.012 & 0.032 & 0.013 & 0.026 \\
\hline & $\mathrm{BE}$ & MSE & 0.189 & 0.148 & 0.351 & 0.074 & 0.087 & 0.195 & 0.312 & 0.467 \\
\hline & & SE & 0.005 & 0.026 & $9.600^{*}$ & 0.034 & $1.500^{*}$ & 0.044 & 0.011 & 0.013 \\
\hline & & MC Error & $2.248^{*}$ & 0.012 & $4.241^{*}$ & 0.015 & $0.584^{*}$ & 0.020 & $4.752^{*}$ & $5.490^{*}$ \\
\hline \multirow[t]{9}{*}{30} & ML & MSE & 0.440 & 0.542 & 0.045 & 0.128 & 0.202 & 0.502 & 0.142 & 0.468 \\
\hline & & SE & 0.021 & 0.023 & $6.217^{*}$ & 0.011 & 0.015 & 0.022 & 0.011 & 0.022 \\
\hline & LS & MSE & 0.038 & 0.263 & 0.038 & 0.133 & 0.040 & 0.365 & 0.065 & 0.306 \\
\hline & & SE & $6.266^{*}$ & 0.015 & $5.420^{*}$ & $8.263^{*}$ & $6.162^{*}$ & 0.017 & $7.244^{*}$ & 0.014 \\
\hline & WLS & MSE & 0.037 & 0.275 & 0.048 & 0.136 & 0.046 & 0.382 & 0.071 & 0.313 \\
\hline & & SE & $6.249^{*}$ & 0.016 & $6.161^{*}$ & $8.230^{\star}$ & $6.820^{*}$ & 0.018 & $7.625^{\star}$ & 0.014 \\
\hline & $\mathrm{BE}$ & MSE & 0.159 & 0.020 & 0.296 & 0.045 & 0.062 & $7.870^{\star}$ & 0.226 & 0.240 \\
\hline & & SE & 0.004 & 0.008 & 0.006 & 0.014 & $0.500^{*}$ & $7.100^{*}$ & $5.200^{*}$ & $9.200^{*}$ \\
\hline & & MC Error & $0.165^{\star}$ & $4.424^{*}$ & $3.447^{*}$ & $7.548^{*}$ & $0.182^{*}$ & $3.843^{*}$ & $2.711^{*}$ & $4.907^{\star}$ \\
\hline \multirow[t]{9}{*}{50} & ML & MSE & 0.218 & 0.335 & 0.037 & 0.097 & 0.149 & 0.307 & 0.079 & 0.354 \\
\hline & & SE & $9.118^{*}$ & 0.011 & $3.151^{*}$ & $4.681^{*}$ & $7.665^{\star}$ & 0.010 & $4.680^{*}$ & 0.010 \\
\hline & LS & MSE & 0.019 & 0.172 & 0.036 & 0.122 & 0.031 & 0.230 & 0.051 & 0.279 \\
\hline & & SE & $2.584^{*}$ & $7.505^{\star}$ & $3.062^{*}$ & $3.906^{*}$ & $3.067^{*}$ & $8.328^{*}$ & $3.631^{*}$ & $6.461^{*}$ \\
\hline & WLS & MSE & 0.021 & 0.173 & 0.045 & 0.136 & 0.031 & 0.226 & 0.052 & 0.268 \\
\hline & & SE & $2.718^{\star}$ & $7.498^{*}$ & $3.338^{*}$ & $4.073^{*}$ & $3.164^{*}$ & $8.274^{*}$ & $3.712^{*}$ & $6.812^{*}$ \\
\hline & $\mathrm{BE}$ & MSE & 0.022 & $6.570^{*}$ & 0.119 & $3.730^{*}$ & $0.370^{*}$ & $0.260^{*}$ & 0.083 & 0.033 \\
\hline & & SE & $0.300^{*}$ & 0.009 & 0.004 & $6.500^{*}$ & $0.200^{*}$ & $2.400^{*}$ & $2.800^{*}$ & $1.400^{*}$ \\
\hline & & MC Error & $0.163^{*}$ & $6.632^{*}$ & $2.937^{*}$ & $4.585^{\star}$ & $0.080^{*}$ & $1.638^{*}$ & $1.898^{*}$ & $9.899^{\star}$ \\
\hline
\end{tabular}

Note: * Indicates that the value is multiplied by $10^{-3}$ 


\section{ON THE EXTENSION OF EXPONENTIATED PARETO DISTRIBUTION}

Table 2. MSEs, SEs, and MC errors of EGEP estimates for Case III and Case IV

\begin{tabular}{|c|c|c|c|c|c|c|c|c|c|c|}
\hline \multirow[b]{2}{*}{$n$} & \multirow[b]{2}{*}{ Method } & \multirow[b]{2}{*}{ Properties } & \multicolumn{4}{|c|}{ Case III } & \multicolumn{4}{|c|}{ Case IV } \\
\hline & & & $\alpha=1$ & $\lambda=2.6$ & $\theta=2$ & $\beta=0.75$ & $\alpha=0.4$ & $\lambda=0.9$ & $\theta=0.7$ & $\beta=0.75$ \\
\hline \multirow[t]{9}{*}{10} & $\mathrm{ML}$ & MSE & 0.164 & 1.142 & 2.615 & 2.673 & 1.259 & 1.066 & 0.542 & 1.176 \\
\hline & & SE & 0.040 & 0.087 & 0.158 & 0.145 & 0.106 & 0.095 & 0.073 & 0.086 \\
\hline & LS & MSE & 0.145 & 0.928 & 2.020 & 1.278 & 0.041 & 0.673 & 0.132 & 0.692 \\
\hline & & SE & 0.033 & 0.074 & 0.139 & 0.108 & 0.018 & 0.067 & 0.029 & 0.077 \\
\hline & WLS & MSE & 0.145 & 0.905 & 2.232 & 1.269 & 0.060 & 0.854 & 0.136 & 0.746 \\
\hline & & SE & 0.033 & 0.071 & 0.148 & 0.106 & 0.023 & 0.076 & 0.029 & 0.080 \\
\hline & BE & MSE & 0.612 & 1.051 & 0.686 & 0.979 & 0.341 & 0.452 & 0.381 & 0.325 \\
\hline & & SE & $5.850^{*}$ & 0.206 & 0.231 & 0.027 & $8.900^{*}$ & 0.055 & 0.018 & 0.018 \\
\hline & & MC Error & $1.329^{*}$ & $8.162^{*}$ & 0.065 & 0.073 & $2.661^{*}$ & 0.017 & $5.438^{*}$ & $5.363^{*}$ \\
\hline \multirow[t]{9}{*}{20} & $\mathrm{ML}$ & MSE & 0.060 & 0.521 & 1.480 & 0.840 & 0.955 & 0.735 & 0.195 & 0.591 \\
\hline & & SE & 0.012 & 0.030 & 0.060 & 0.041 & 0.047 & 0.040 & 0.022 & 0.031 \\
\hline & LS & MSE & 0.094 & 0.537 & 1.081 & 0.604 & 0.023 & 0.494 & 0.090 & 0.250 \\
\hline & & SE & 0.011 & 0.022 & 0.052 & 0.037 & $6.063^{*}$ & 0.026 & $9.708^{*}$ & 0.021 \\
\hline & WLS & MSE & 0.085 & 0.525 & 1.113 & 0.668 & 0.046 & 0.705 & 0.079 & 0.319 \\
\hline & & SE & 0.011 & 0.022 & 0.053 & 0.039 & 0.010 & 0.034 & $9.380^{*}$ & 0.024 \\
\hline & BE & MSE & 0.579 & 0.329 & 0.142 & 0.923 & 0.268 & 0.257 & 0.281 & 0.237 \\
\hline & & SE & 0.016 & 0.117 & 0.076 & 0.034 & 0.010 & 0.043 & 0.021 & 0.011 \\
\hline & & MC Error & $6.806^{*}$ & 0.015 & 0.052 & 0.034 & $4.546^{\star}$ & 0.019 & $9.202^{*}$ & $4.719^{*}$ \\
\hline \multirow[t]{9}{*}{30} & $\mathrm{ML}$ & MSE & 0.041 & 0.342 & 1.290 & 0.590 & 0.310 & 0.497 & 0.123 & 0.293 \\
\hline & & SE & $6.466^{*}$ & 0.017 & 0.038 & 0.023 & 0.018 & 0.022 & 0.011 & 0.014 \\
\hline & LS & MSE & 0.083 & 0.476 & 0.788 & 0.427 & 0.021 & 0.454 & 0.090 & 0.199 \\
\hline & & SE & $5.914^{*}$ & 0.013 & 0.029 & 0.021 & $3.654^{*}$ & 0.016 & $5.835^{\star}$ & 0.012 \\
\hline & WLS & MSE & 0.069 & 0.445 & 0.632 & 0.789 & 0.039 & 0.694 & 0.076 & 0.208 \\
\hline & & SE & $5.270^{*}$ & 0.012 & 0.027 & 0.028 & $5.895^{\star}$ & 0.021 & $5.669^{*}$ & $9.454^{*}$ \\
\hline & $B E$ & MSE & 0.039 & 0.473 & 0.087 & 0.793 & 0.135 & 0.034 & 0.086 & 0.037 \\
\hline & & SE & $1.297^{*}$ & 0.045 & 0.031 & 0.019 & $0.800^{*}$ & 0.021 & 0.011 & $7.800^{*}$ \\
\hline & & MC Error & $0.373^{*}$ & 0.024 & 0.017 & 0.011 & $0.368^{*}$ & 0.012 & $6.042^{*}$ & $4.252^{*}$ \\
\hline \multirow[t]{9}{*}{50} & $M L$ & MSE & 0.041 & 0.298 & 1.305 & 0.522 & 0.223 & 0.347 & 0.067 & 0.180 \\
\hline & & SE & $3.698^{*}$ & $9.216^{*}$ & 0.023 & 0.013 & $9.185^{*}$ & 0.011 & $4.786^{*}$ & $6.670^{*}$ \\
\hline & LS & MSE & 0.069 & 0.442 & 0.623 & 0.575 & 0.023 & 0.395 & 0.077 & 0.157 \\
\hline & & SE & $2.801^{*}$ & $5.658^{*}$ & 0.015 & 0.015 & $2.471^{*}$ & $9.006^{*}$ & $3.113^{*}$ & $5.953^{*}$ \\
\hline & WLS & MSE & 0.054 & 0.381 & 0.432 & 0.298 & 0.023 & 0.576 & 0.065 & 0.196 \\
\hline & & SE & $2.504^{*}$ & $5.256^{*}$ & 0.014 & 0.010 & $2.541^{*}$ & 0.012 & $3.197^{*}$ & $7.415^{\star}$ \\
\hline & $\mathrm{BE}$ & MSE & 0.037 & 0.431 & 0.049 & 0.784 & $9.860^{*}$ & $5.660^{*}$ & 0.014 & $2.890^{*}$ \\
\hline & & SE & $3.499^{*}$ & 0.090 & 0.030 & $9.603^{*}$ & $0.200^{*}$ & $9.400^{*}$ & $6.200^{*}$ & $7.300^{*}$ \\
\hline & & MC Error & $2.228^{*}$ & 0.064 & 0.021 & $6.768^{*}$ & $0.896^{*}$ & $6.633^{*}$ & $4.341^{*}$ & $5.192^{*}$ \\
\hline
\end{tabular}

Note: * Indicates that the value is multiplied by $10^{-3}$ 


\section{HASSAN ET AL}

The following conclusions can be detected about the performance of different estimates:

- In all cases, the MSEs of Bayesian estimates are better than MSEs of ML, LS, and WLS estimates (see Tables 1 and 2).

- For all methods of estimations, it is clear that MSEs, SEs, and MC error decrease as sample size increases (see Tables 1 and 2).

- The MSEs and SEs of LS estimates, for all parameters values, are the smallest among the other estimates in almost all of the cases (see for example Table 1).

- For fixed value of $\lambda=0.7, \theta=0.25$ and as the value of shape parameters $\alpha, \beta$ increases, the MSEs and SEs for estimates $\lambda, \theta$ are increasing based on ML, LS and WLS methods but decreasing based on BE. Also, the MSEs and SEs for estimates $\alpha$, are decreasing based on ML and Bayesian methods but increasing based on LS and WLS methods. In addition, the MSEs and SEs for estimates are increasing based on ML, LS, and WLS methods while decreasing based on BE (see Table 1).

- When the values of $\alpha, \theta$, and $\lambda$ increase and the shape parameter $\beta$ decrease, the MSEs and SEs for estimates of the unknown parameters based on ML, LS, WLS, and BE methods are increasing in approximately most of situations (see Tables 1 and 2).

- For the Case III of parameters $(\alpha=1, \lambda=2.6, \theta=2, \beta=0.75)$ the Bayesian estimates have good statistical properties than the other cases of parameters (see Tables 1 and 2).

\section{Application}

We provide an application to real data set to illustrate the importance and flexibility of the EGEP distribution. The real data represent the survival times, in weeks, of 33 patients suffering from acute Myelogeneous Leukaemia. The data were analyzed by Feigl and Zelen (1965). The data are: 65, 156, 100, 134, 16, 108, 121, 4, 39, 143, $56,26,22,1,1,5,65,56,65,17,7,16,22,3,4,2,3,8,4,3,30,4,43$.

We compare the fit of EGEP distribution to sub-models; EP and SP distributions; with competitive model, namely, additive Weibull (AW) (Almalki \& Yuan, 2013).

In order to compare the models, we consider some goodness-of-fit measures including: $-2 L$ where $L$ is the maximized log-likelihood, Akaike information criterion (AIC), Bayesian information criterion (BIC), and the corrected Akaike 


\section{ON THE EXTENSION OF EXPONENTIATED PARETO DISTRIBUTION}

information criterion (CAIC). Generally, the smaller values of these statistics, the better fit to real data. Additionally, the histogram plots and the estimated pdf of the models for the data set are shown. Furthermore, the plots of empirical cdf and estimated cdf of models are displayed.

Table 3. ML estimates, $-2 L, A I C, B I C$, and CAIC for acute Myelogeneous Leukaemia data

\begin{tabular}{crrrrr} 
Model & ML estimates & $\mathbf{- 2 L}$ & AIC & BIC & CAIC \\
\hline EGEP & $\alpha=1.000$ & 303.234 & 311.234 & 317.097 & 319.420 \\
& $\beta=4.205$ & & & & \\
& $\theta=1.000$ & & & & \\
AW & $\lambda=0.710$ & & & & \\
& $a=2.003$ & 305.071 & 316.425 & 321.203 & 326.112 \\
& $b=5.642$ & & & & \\
& $c=3.351$ & & & & \\
EP & $d=1.830$ & & & & \\
& $\theta=5.271$ & 323.696 & 325.696 & 327.162 & 327.763 \\
SP & $\lambda=6.032$ & & & & \\
& $\lambda=6.032$ & 394.686 & 398.686 & 401.617 & 402.789 \\
\hline
\end{tabular}
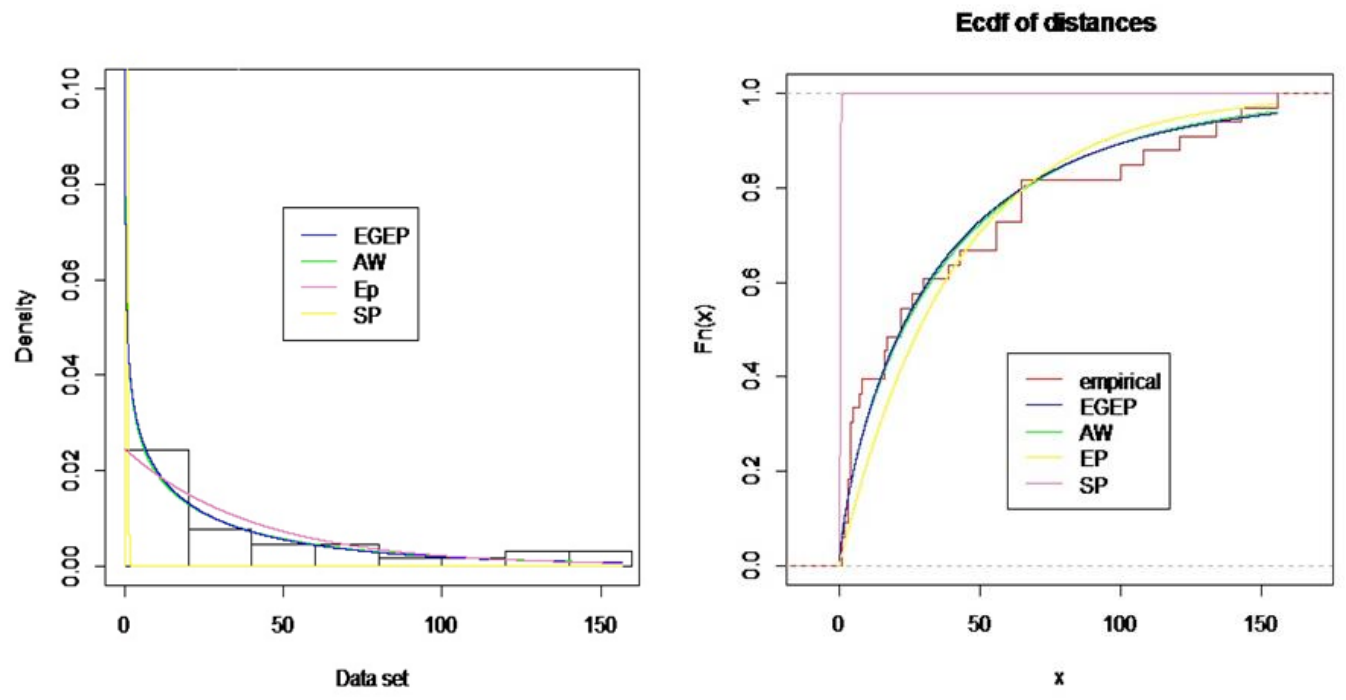

Figure 3. Estimated pdf and cdf of the models for the real data set 


\section{HASSAN ET AL}

In general, the model with minimum values for these statistics could be chosen as the best model to fit the data. Table 3 lists the ML estimates of the model parameters and the values of statistics; $-2 L$, AIC, BIC, and CAIC for the fitted models to the real data set. It is noted from Table 3 that the EGEP distribution gives the lowest values for the $-2 L$, AIC, BIC, and CAIC statistics among all fitted models. Thus, the EGEP distribution could be chosen as the best models.

The results show that the EGEP distribution provides a significantly better fit than the other three models. Plots of the estimated pdf and cdf of the EGEP, AW, EP, and SP models fitted to this data set are given in Figure 3. The figures indicate that the EGEP distribution is superior to the other distributions in terms of model fitting.

\section{Concluding Remarks}

We introduce and study a new lifetime distribution, the so-called the exponentiated generalized exponentiated Pareto distribution as a generalization of the Pareto and the exponentiated Pareto distributions. A detailed study on the statistical properties of the new distribution is presented. The ordinary moments, Rényi entropy and moments of residual are derived. Estimation of parameters is approached by maximum likelihood, least squares, weighted least squares and Bayesian methods. A simulation study is implemented for investigating the accuracy of different estimates for different sample sizes. An application to real data shows the superiority of the proposed model than some other models.

\section{Acknowledgements}

The authors are grateful to the editor and referees for their constructive comments and valuable suggestions which certainly improved the presentation and quality of the article.

\section{References}

Abdul-Moniem, I. B., \& Abdel-Hameed, H. F. (2012). On exponentiated Lomax distribution. International Journal of Mathematical Education, 3(5), 1-7.

Alexander, C., Cordeiro, G. M., Ortega, E. M. M., \& Sarabia, J. M. (2012). Generalized beta-generated distributions. Computational Statistics \& Data Analysis, 56(6), 1880-1897. doi: 10.1016/j.csda.2011.11.015 


\section{ON THE EXTENSION OF EXPONENTIATED PARETO DISTRIBUTION}

Alizadeh, M., Altun, E., Afify, A. Z., \& Ozel, G. (2019). The extended odd Weibull-G family: Properties and applications. Communications Faculty of Sciences University of Ankara Series A1 Mathematics \& Statistics, 68(1), 161186. Retrieved from https://dergipark.org.tr/en/pub/cfsuasmas/article/443699

Almalki, S. J., \& Yuan, J. (2013). A new modified Weibull distribution. Reliability Engineering and System Safety, 111, 164-170. doi:

10.1016/j.ress.2012.10.018

Alzaatreh, A., Lee, C., \& Famoye, F. (2013). A new method for generating families of continuous distributions. METRON, 71, 63-79. doi: 10.1007/s40300013-0007-y

Alzaghal, A., Famoye, F., \& Lee, C. (2013). Exponentiated $T$-X family of distributions with some applications. International Journal of Statistics and Probability, 2(3), 31-49.

Bourguignon, M., Silva, R. B., \& Cordeiro, G. M. (2014). The Weibull-G family of probability distributions. Journal of Data Science, 12(1), 53-68.

Cordeiro, G. M., Afify, A. Z., Ortega, E. M., Suzuki, A. K., \& Mead, M. E. (2019). The odd Lomax generator of distributions: Properties, estimation and applications. Journal of Computational and Applied Mathematics, 347, 222-237. doi: 10.1016/j.cam.2018.08.008

Cordeiro, G. M., Alizadeh, M., \& Diniz, P. R. (2015). The type I halflogistic family of distributions. Journal of Statistical Computation and Simulation, 86(4), 707-728. doi: 10.1080/00949655.2015.1031233

Cordeiro, G. M., Alizadeh, M., Ozel, G., Hosseini, B., Ortega, E. M. M., \& Altun, E. (2017). The generalized odd log-logistic family of distributions: properties, regression models and applications. Journal of Statistical Computation and Simulation, 87(5), 908-932. doi: 10.1080/00949655.2016.1238088

Cordeiro, G. M., \& de Castro, M. (2011). A new family of generalized distributions. Journal of Statistical Computation and Simulation, 81(7), 883-893. doi: 10.1080/00949650903530745

Cordeiro, G. M., Ortega, E. M. M., \& da Cunha, D. C. C. (2013). The exponentiated generalized class of distributions. Journal of Data Science, 11(1), $1-27$.

Cordeiro, G. M., Ortega, E. M. M., \& Nadarajah, S. (2010).The Kumaraswamy Weibull distribution with application to failure data. Journal of the Franklin Institute, 347(8), 1399-1429. doi: 10.1016/j.jfranklin.2010.06.010 


\section{HASSAN ET AL}

El-Sherpieny, E. A., \& Ahmed, M. A. (2014). On the Kumaraswamy Kumaraswamy distribution. International Journal of Basic and Applied Sciences, 3(4), 372-381.

Eugene, N., Lee, C., \& Famoye, F. (2002). Beta-normal distribution and its applications. Communication in Statistics - Theory and Methods, 31(4), 497-512. doi: 10.1081/STA-120003130

Feigl, P., \& Zelen, M. (1965). Estimation of exponential probabilities with concomitant information. Biometrics, 21(4), 826-838. doi: 10.2307/2528247

Gupta, R. C., Gupta, R. D., \& Gupta, P, L. (1998). Modeling failure time data by Lehman alternatives. Communications in Statistics - Theory and Methods, 27(4), 887-904. doi: 10.1080/03610929808832134

Gupta, R. D., \& Kundu, D. (1999). Generalized exponential distribution. Australian \& New Zealand Journal of Statistics, 41(2), 173-188. doi: 10.1111/1467-842X.00072

Hassan, A. S., \& Elgarhy, M. (2016a). A new family of exponentiated Weibull-generated distributions. International Journal of Mathematics and its Applications, 4(1-D), 135-148. Retrieved from http://ijmaa.in/v4n1-d/135-148.pdf

Hassan, A. S., \& Elgarhy, M. (2016b). Kumaraswamy Weibull-generated family of distributions with applications. Advances and Applications in Statistics, 48(3), 205-239. doi: 10.17654/AS048030205

Hassan, A. S., Elgarhy, M., Ahmad, Z. (2019). Type II Generalized ToppLeone family of distributions: Properties and applications. Journal of Data Sciences, 17(4), 638-658. doi: 10.6339/JDS.201910_17(4).0001

Hassan, A. S., Elgarhy, M., \& Shakil, M. (2017). Type II half logistic family of distributions with applications. Pakistan Journal of Statistics and Operation Research, 13(2), 245-264. doi: 10.18187/pjsor.v13i2.1560

Hassan, A. S., \& Hemeda, S. E. (2016). The additive Weibull-G family of probability distributions. International Journal of Mathematics and its Applications, 4(2-A), 151-164. Retrieved from http://ijmaa.in/v4n2-a/151-164.pdf

Hassan, A. S., Hemeda, S. E., Maiti, S.S., \& Pramanik, S. (2017). The generalized additive Weibull-G family of probability distributions. International Journal of Statistics and Probability, 6(5), 65-83.

Hassan, A. S. \& Nassr, S. G. (2018a). The inverse Weibull generator of distributions: Properties and applications. Journal of Data Sciences, 16(4), 732742. doi: 10.6339/JDS.201810_16(4).00004 


\section{ON THE EXTENSION OF EXPONENTIATED PARETO DISTRIBUTION}

Hassan, A. S., \& Nassr, S. G. (2018b). Power Lindley-G family of distributions. Annals of Data Science, 6(2), 189-210. doi: 10.1007/s40745-0180159-y

Johnson, N. L., Kotz, S., \& Balakrishnan, N. (1995). Continuous univariate distribution ( $2^{\text {nd }}$ edition). New York: Wiley and Sons.

Jones, M. C. (2004). Families of distributions arising from the distributions of order statistics. Test, 13(1), 1-43. doi: 10.1007/BF02602999

Lemonte, A. J., Souza, W. B., \& Cordeiro, G. M. (2013). The exponentiated Kumaraswamy distribution and its log-transform. Brazilian Journal of Probability and Statistics, 27(1), 31-53. doi: 10.1214/11-BJPS149

Lomax, H. S. (1954). Business failures: Another example of the analysis of failure data. Journal of the American Statistical Association, 49(268), 847-852. doi: 10.1080/01621459.1954.10501239

Marshall, A. N., \& Olkin, I. (1997). A new method for adding a parameter to a family of distributions with applications to the exponential and Weibull families. Biometrika, 84(3), 641-552. doi: 10.1093/biomet/84.3.641

Navarro, J., Franco, M., \& Ruiz, J. M. (1998). Characterization through moments of the residual life and conditional spacings. Sankhyā Series A, 60(1), 36-48.

Rodrigues, J. A., \& Silva, A. P. C. M. (2015). The exponentiated Kumaraswamy exponential distribution. British Journal of Applied Science \& Technology, 10(1), 1-12.

Silva, F. G., Percontini, A., de Brito, E., Ramos, M. W., Venancio, R., \& Cordeiro, G. M. (2017). The odd Lindley-G family of distributions. Austrian Journal of Statistics, 46(1), 65-87. doi: 10.17713/ajs.v46i1.222

Tahir, M. H., Cordeiro, G. M., Alzaatreh, A., Zubair, M., \& Mansoor, M. (2016). The logistic-X family of distributions and its applications. Communications in Statistics - Theory \& Methods, 45(24), 7326-7349. doi: 10.1080/03610926.2014.980516

Zografos, K., \& Balakrishnan, N. (2009). On families of beta-and generalized gamma-generated distributions and associated inference. Statistical Methodology, 6(4), 344-362. doi: 10.1016/j.stamet.2008.12.003 